\title{
Design and Realization of Life-cycle cost Calculation Modularization -Based on Percolation Theory
}

\author{
Yujing Wang ${ }^{1, a}$, Weiyi $\mathrm{He}^{1, \mathrm{~b}}$ \\ ${ }^{1}$ School of Tianjin University of Technology, Tianjin 300000, China; \\ a1510423135@qq.com, bweiyihe5@126.com
}

Keywords: Life-cycle cost, Modularization, Technical secondary development, Percolation theory, Platform design.

\begin{abstract}
Inorder to improve the life-cycle cost analysis (LCC) in the project of large-scale use of a flexible system, the introduction of "percolation theory" was used and proposed LCC calculation module system consists of basic modules, function modules, the module structure of three modules, and It was confirmed through the implementation of example.
\end{abstract}

\section{Introduction}

Engineering design innovation project cost is usually subject to economic constraints, only through the life-cycle cost analysis (LCC) can we reveal the economic value of sustainable design or green design ${ }^{[1]}$. But the biggest problem of LCC engineering applications is insufficient data supply, in order to ensure the reliability of data and validity of estimation results, calculation models must be secondary development. The development is difficult and the development process can't be copied, which caused high use-cost, and seriously hindered LCC-scale use in the design activities.

\section{Accounting issues and modular target of LCC}

LCC design process is generally regarded as the later technical and economic evaluation activities. However, due to differences in engineering backgrounds, different calculating demands, insufficient supply of data resources, resulting in the secondary development of calculating model rather than direct selection when every engineering application ${ }^{[2]}$, difficult and inefficient, is a complex system problem to cope with for technical and economic professionals (such as cost engineer $)^{[3]}$. The current domestic and international researches on LCC engineering applications are mainly focused on the design of LCC calculation model development considering different professional characteristics and module goals, research and development as the main researchers in universities. The modular study for the purpose of Engineering scale implementation to solve complexity development process of the LCC calculation model has not been found. This paper argues that under the double constraints limit in technology and economy, the maximum to meet the needs of the perpetrators of LCC maximize meet the needs of LCC implementers, is the modular design goals of LCC calculation process.

\section{Module system design of LCC calculation process}

In view of the overall requirements of the project design of complex composition and quality and schedule management, this study is based on module partition of product ideas, consider three custom dimension constraints: professional engineering background, the utility of LCC estimation and its supporting data. The LCC measurement model development process is divided into basic module, function module, structure module, referred to as the LCC measurement model development module. Base module solve the problem of the application of LCC professional scene and the function module and the structure module through the interface rule, opens the forecast technology and the supporting data resources tight coupling, the specific meaning of each module are as follows:

Base module: Design is a prerequisite to achieve LCC consulting and is a universal carrier of 
the module, this study set the base module. Specific role is to identify the cost breakdown structure cost factors. In order to response the real needs of the LCC advisory, the partitioning of the base module must be consistent with the general engineering breakdown structure.

Function module: In the specific engineering situation of the LCC calculation function needs, The calculation model must satisfy the structural model rules, the study is set as a function module. The specific role is to complete of a certain validity of the cost estimates of the cost factors modeling and estimating . In order to response the real needs of the LCC advisory, the partition of the function module must match the different calculation validity.

Structure module: In the context of the specific basic module, the coordination and convergence of the function of LCC measurement, calculation model, data resources, information technology and other elements must be considered, reasonable division and coordination of between the three interface module and between the internal sub module interface need certain rules to be standardized and standardized, this study set the structure module, The specific role is to provide the LCC module configuration rules, the basic module and the function module connect together, structure module determines types and combinations of base modules and function modules to be loaded.

\section{LCC calculation modular platform design Based on the "percolation theory"}

"Percolation theory" thought, in a huge disordered system, when the increases or decreases of the degree, density and concentration of media interconnected reach a certain level, the system has the potential to change the nature of the mutation or acute phase change, such as a blockage / circulation, curb / pop, retardant / diffusion, designate "percolation transition."

In the process of "percolation", medium control fluid movement ${ }^{[4]}$. Refer to the "percolation theory", this paper proposes that during the LCC consulting service process, its modular system belongs to a controllable retardant medium, only if calculation model development capabilities and ability to obtain data combination controllable situation, LCC Consulting Services state will transform from retardant to diffusion. Thus, only by building a service platform, the data module is integrated into the resource estimation model development module system, the module of data resources are integrated into the calculation model development module system, to control the matching degree of measure model development capabilities and data acquisition capabilities, can eventually realize the modular performance targets.

In this paper, take municipal engineering for example, design the "Municipal Engineering LCC Calculation Modular Platform", as the subsystem of the LCC consulting service platform, its outstanding feature is data resource module embedded in the LCC calculation model secondary development module system, during Municipal engineering operation period maintenance costs and calculation process has been modularized based on different data resources condition.

The design logic of this platform is shown in Fig 1. 


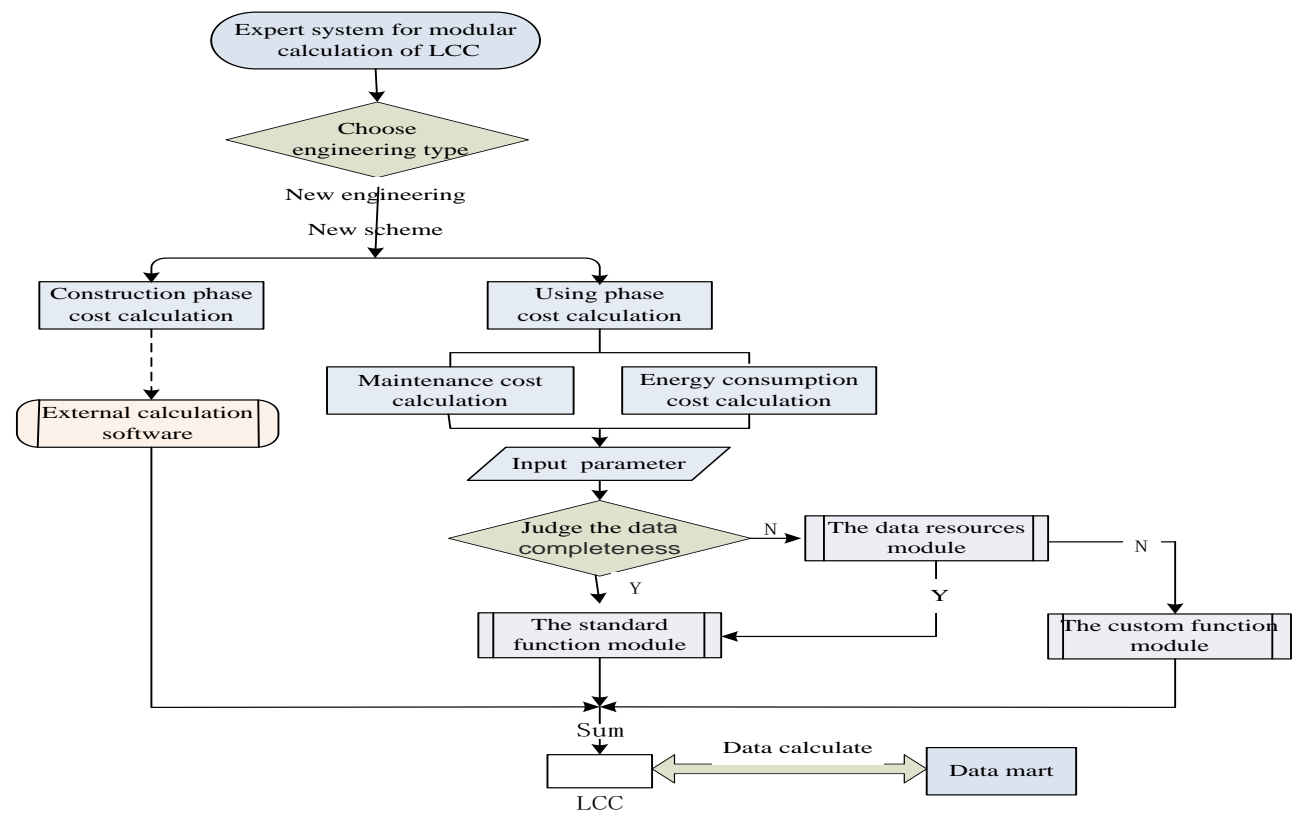

Fig 1. Architecture of Municipal Engineering LCC Calculation Modular Platform

According to "percolation theory", the platform designed two calculation function modules: universal function modules and custom function modules, and there are three custom solutions: recommend function module, development function module and data function module. The system recommend the user to input other relevant data in case lacking of calculation data, click into the recommended function, the recommended functional modules provides different calculation model paths, the user can choose according to their own data resources. Development functional module is a LCC modeling tools providing the users special needs. The user can use the data list and graphics automatically generating from platform to compare the LCC and child program of schemes, and then select the optimal scheme. Data module is recommended to use when the user own a small amount of data. Data resources can be queried and got through system recommend path, and then complete the LCC calculation on the platform. The data module depends on the database platform construction, so the measurable transaction data resources trading can be used to provide high-value LCC consultation services for platform using units or departments and their customers.

\section{Application examples}

This study has been carried out simulation run "Municipal Engineering LCC Calculation Modular Platform" cooperative development with Tianjin University. This platform mainly take SSH (Struts, Spring, Hibernate) as framework, Liferay as interface design, Eclipse as development environment, java as development language, MySQL as the backend database management system operation platform to store data and resources.

Take the choice of municipal engineering asphalt pavement structure scheme as an example, firstly, the system can trigger an external calculate software (such as the Glodon cost calculation software) through the interface to complete cost calculation. Next, the system can calculate the present maintenance costs of maintenance schemes based on different asphalt thickness parameter in different schemes input by users. It can also calculate the energy costs of the pavement through recommend function subsystem and data module subsystem when lacking of enough data to use. Users can quickly find a suitable calculation model through the system prompt, and then trigger backend function modules subsystem to calculate, and finally provide intuitive consulting results on the user interface.

\section{Conclusion}

This paper presents a modular architecture designed to promote LCC technology scale 
applications in engineering, designs LCC calculation module system and modular platform which integrates the data module. And take municipal engineering for example to verify the practicality and efficiency of this platform. This study contributes to improve flexibility of LCC technology, prompts engineering cost professional to adapt LCC technology, thus promoting the engineering design innovation.

\section{Acknowledgement}

This study was supported by 2015 Annual Tianjin Philosophy and Social Sciences Planning Program (TJGL15-005)

\section{References}

[1] Kshirsagar A S, El-Gafy M A: Suitability of life cycle cost analysis (LCCA) as asset management tools for institutional buildings. Journal of Facilities Management, Vol.8 (2010), No3, p162.

[2] Newnes L B: Predicting the Whole-Life Cost of a product at the conceptual design stage Journal of Engineering Design, Vol.19 (2008) No.2, p99.

[3] Olubodun F, Kangwa J, Oladapo A: An appraisal of the level of application of life cycle costing within the construction industry in the UK Structural Survey, Vol.28 (2010) No.4, p254-265.

[4] Liu Shengli, Feng Huixia: Research and application of percolation theory Journal of Applied Chemical Industry Vol.39 (2010) No.7, p1074-1078. 